\title{
Efecto de la succión en la estabilidad de un talud de arena limosa
}

\author{
Effect of the Suction on the Stability of a Silty Sand Slope
}

\author{
Gallegos-Fonseca G. \\ División de Estudios de Posgrado \\ Facultad de Ingeniería \\ Universidad Autónoma de Querétaro \\ Correo: gfonseca@uaslp.mx \\ Leal-Vaca J.C. \\ División de Estudios de Posgrado \\ Facultad de Ingeniería \\ Universidad Autónoma de Querétaro \\ Correo: jcesarlealv@hotmail.com
}

\author{
Rojas-González E. \\ Facultad de Ingeniería \\ División de Estudios de Posgrado \\ Universidad Autónoma de Querétaro \\ Correo:erg@uaq.mx \\ Mora-Ortíz R.S. \\ Facultad de Ingeniería \\ División de Estudios de Posgrado \\ Universidad Autónoma de Querétaro \\ Correo: renemora1221@hotmail.com
}

Información del artículo: recibido: diciembre de 2008, reevaluado: junio de 2010, aceptado: junio de 2011

\section{Resumen}

En este trabajo se presenta la revisión de algunos modelos que se han propuesto para estimar la resistencia al cortante de los suelos no saturados. Los resultados teóricos obtenidos mediante estos modelos se comparan con los resultados de una serie de ensayes triaxiales a succión controlada, realizados sobre un suelo areno limoso con diferentes valores de succión. De esta comparación se seleccionó el modelo que mejor predice el comportamiento de la resistencia de este tipo de suelo. Se hace una aplicación real del modelo seleccionado empleándolo en el análisis de la estabilidad de un talud de arcilla de alta compresibilidad, sujeta a cambios en su grado de saturación. Se sabe que el grado de saturación de un suelo está directamente relacionado con la succión y que la succión es una variable independiente de los esfuerzos que influye en la resistencia de los suelos no saturados. Por lo tanto, al modificarse el contenido de agua de un talud también lo hace su factor de seguridad. Este ejercicio permite obtener algunas conclusiones importantes con respecto al factor de seguridad de los taludes.

\section{Descriptores}

- succión

- estabilidad de taludes

- esfuerzos de corte

- esfuerzos efectivos

- trayectoria de humedecimiento 


\begin{abstract}
This paper presents a revision of some of the models that have been proposed to estimate the strength of unsaturated soils. The theoretical results obtained from these models are compared with the experimental results of controlled suction triaxial tests made on silty sand. The model that resulted in the best prediction was used to determine the stability of a slope of highly compressive clay subjected to changes in its water content. It is well known that the water content of soil is directly related with suction and that suction is an independent variable of the stress state that modifies the shear strength of unsaturated soils. Therefore, when the water content of a slope is modified, the safety factor of the slope is also modified. This exercise allows drawing some important conclusions over the stability of slopes related to the safety factor.
\end{abstract}
Keywords
- suction
- slope stability
- strength stress
- effective stress
- wetted path

\section{Introducción}

Un talud es toda superficie del suelo que presenta cierta inclinación con respecto a la horizontal y puede ser natural o artificial. Algunas de estas superficies tienden a deslizarse debido a la acción de diversos agentes como la gravedad, cambios en su contenido de agua o por modificaciones en su geometría. La geometría del talud, la resistencia al esfuerzo cortante del suelo, el intemperismo, el drenaje y la vegetación son algunos aspectos que afectan su estabilidad.

Es muy frecuente que se presente el deslizamiento de taludes durante el periodo de lluvias. Las fallas en los taludes se pueden presentar de manera repentina, debido a la reducción rápida de la resistencia al esfuerzo cortante, provocada por el incremento en el contenido de agua del suelo durante un periodo de lluvias intensas.

Adicionalmente, se sabe que el cambio climático está provocando lluvias más intensas y prolongadas en algunas zonas del planeta, propiciando cambios en las condiciones de estabilidad de algunos taludes que habían permanecido estables durante muchos años.

Por lo antes expuesto, es importante evaluar las variaciones del factor de seguridad de aquellos taludes localizados en zonas habitacionales, industriales, carreteras y en todos aquellos sitios en los cuales se puedan poner en riesgo vidas humanas y ocasionar pérdidas económicas.

En los suelos no saturados, la presencia de los meniscos de agua induce fuerzas de contacto adicionales entre las partículas sólidas que incrementan su resistencia. Por lo tanto, la reducción de la resistencia que experimentan estos suelos se debe al decremento de los esfuerzos de succión en el suelo, ocasionados por la desaparición de los meniscos del agua de los poros al momento en el que se presenta la infiltración del agua de lluvia.

La succión total en un suelo no saturado la constituyen la succión mátrica (producida por los meniscos) y la succión osmótica (producida por las sales disueltas en el agua de poro). En un suelo no saturado, las variables independientes de los esfuerzos que influyen en su resistencia son el esfuerzo neto $\left(\sigma_{n}-u_{a}\right)$ y la succión $\left(u_{a}-u_{w}\right)$; en donde $\sigma_{\mathrm{n}}$ representa el esfuerzo normal total, $u_{a}$ la presión del aire y $u_{w}$ la presión del agua (Fredlund y Rahardjo, 1993). Actualmente se sabe que la curva característica encierra información valiosa para estimar los esfuerzos de corte, la conductividad hidráulica, los cambios de volumen, etcétera, en los suelos no saturados (Vanapalli et al., 1996). De modo que conocer cómo se encuentra distribuida el agua dentro de los poros del suelo ayudará a comprender mejor el comportamiento de estos materiales (Rojas et al., 2006).

En la literatura especializada existen distintas propuestas para predecir la resistencia de los suelos, por ejemplo: Coulomb (1776), Terzaghi (1936), Bishop (1959), Fredlund et al. (1978) y Vanapalli et al. (1996); estos modelos se describen a continuación. En este trabajo se busca seleccionar el modelo teórico que mejor se apegue a los resultados experimentales, tomando en cuenta que el suelo en análisis exhibe una resistencia al esfuerzo cortante que se atribuye a la cohesión entre las partículas finas, esta es la razón por la cual este modelo también puede aplicarse a otros suelos cohesivos, como es el caso de las arcillas de alta compresibilidad $\mathrm{CH}$.

En la primera parte de esta investigación se presentan los resultados de un programa experimental para conocer la variación de la resistencia al esfuerzo cortante de un suelo, provocado por la variación en su grado de saturación. El programa experimental consistió en desarrollar una serie de ensayes triaxiales (CD) bajo 
succión controlada, en trayectoria de secado y humedecimiento para un esfuerzo isotrópico de $150 \mathrm{kPa}$, practicados a una arena limosa SM. Posteriormente se hace una comparación entre los esfuerzos cortantes obtenidos teóricamente, utilizando los diferentes modelos propuestos con los resultados experimentales, para seleccionar al modelo que mejor reproduzca su comportamiento. En la segunda parte se presenta un ejercicio de aplicación en un talud localizado en la autopista Cuernavaca-Acapulco.

A decir verdad, el suelo del talud no es el mismo que el suelo analizado, el cual sirvió para validar el mejor modelo -en la primera parte-, sin embargo, el efecto del humedecimiento y secado que se presenta en este suelo, es el mismo para cualquier tipo de suelo que presente cohesión.

\section{Descripción de los modelos teóricos de resisten- cia al esfuerzo cortante utilizados}

Los primeros intentos por establecer una teoría de la resistencia al corte de los suelos saturados se atribuyen a Coulomb (1776), quien consideró que ésta se debía a la fricción entre las partículas del suelo y a una liga entre ellas, a la cual llamó cohesión; así planteó el modelo de resistencia para suelos cohesivo-friccionantes.

$\tau=c+\sigma \tan \phi$

donde:

$\tau=$ esfuerzo cortante del suelo

$c=$ cohesión del suelo

$\phi=$ ángulo de fricción interna del material

$\sigma=$ esfuerzo normal total.

Posteriormente, Terzaghi (1936) modificó el modelo de resistencia de Coulomb, mediante el concepto de los esfuerzos efectivos. Dichos esfuerzos están definidos como la diferencia entre el esfuerzo total y la presión del agua de poro, así el nuevo concepto de resistencia para suelos quedó de la siguiente forma:

$\tau=c^{\prime}+\left(\sigma-u_{n}\right) \tan \phi^{\prime}$

donde:

$\tau=$ esfuerzo cortante del suelo

$c^{\prime}=$ cohesión efectiva

$\phi^{\prime}=$ ángulo efectivo de fricción

$u_{n}=$ presión del agua de poro

$\sigma=$ esfuerzo normal total.
Además el término $\left(\sigma-u_{n}\right)$ representa el esfuerzo efectivo aplicado al suelo. Esta es la ecuación de resistencia que se aplica para el caso de suelos saturados, dado que la presión de poro en este caso se considera positiva. Posteriormente, Bishop (1959), planteó que la ecuación de Terzaghi también se puede utilizar para establecer la resistencia al corte en suelos no saturados, sólo que en este caso, la ecuación estaría dada por los parámetros de los esfuerzos efectivos:

$\tau=c^{\prime}+\left(\sigma_{n}-u_{a}\right) \tan \phi^{\prime}+\left(u_{a}-u_{w}\right)(\chi) \tan \phi^{\prime}$

donde:

$\tau=$ esfuerzo cortante del suelo

$c^{\prime}=$ cohesión efectiva

$\sigma_{n}=$ esfuerzo normal total

$u_{a}=$ presión del aire de poro

$u_{w}=$ presión del agua de poro

$\phi^{\prime}=$ ángulo efectivo de fricción

$\chi=$ parámetro que depende del grado de satura ción del suelo (de 0 a 1).

Aquí $\left(\sigma_{n}-u_{a}\right)$ es el esfuerzo neto y $\left(u_{a}-u_{w}\right)$ es la succión mátrica del suelo.

Otra ecuación para predecir la resistencia al corte de los suelos no saturados fue desarrollada por Fredlund et al. (1978), quienes propusieron la relación:

$\tau=c^{\prime}+\left(\sigma_{n}-u_{a}\right) \tan \phi^{\prime}+\left(u_{a}-u_{w}\right) \tan \phi^{b}$

donde:

$\tau=$ esfuerzo cortante del suelo

$c^{\prime}=$ cohesión efectiva

$\sigma_{n}=$ esfuerzo normal total

$u_{a}=$ presión del aire de poro

$u_{w}=$ presión del agua de poro

$\phi^{\prime}=$ ángulo efectivo de fricción

$\phi^{b}=$ ángulo de fricción interna aparente, obtenido de la curva de esfuerzos cortantes contra succión (sus valores son pequeños, debido a que el esfuerzo cortante varía en un orden de magnitud mientras que la succión lo hace en hasta 6 órdenes de magnitud).

También $\left(\sigma_{n}-u_{a}\right)=$ esfuerzo neto y $\left(u_{a}-u_{w}\right)=$ succión mátrica del suelo.

En fechas más recientes han surgido otros modelos que se apoyan en la curva característica del suelo para determinar la magnitud de los esfuerzos de corte como es el caso de Vanapalli et al. (1996), su modelo es: 
$\tau=c^{\prime}+\left(\sigma_{n}-u_{w}\right) \tan \phi^{\prime}+\left(u_{a}-u_{w}\right)\left[\frac{\theta_{w}-\theta_{r}}{\theta_{s}-\theta_{r}}\right] \tan \phi^{\prime}$

donde:

$\tau=$ esfuerzo cortante del suelo

$c^{\prime}=$ cohesión efectiva

$\sigma_{n}=$ esfuerzo normal total

$u_{w}=$ presión del agua de poro

$u_{a}=$ presión del aire de poro

$\phi^{\prime}=$ ángulo efectivo de fricción

$\theta_{w}=$ contenido volumétrico de agua

$\theta_{\mathrm{s}}=$ contenido volumétrico de agua saturado

$\theta_{\mathrm{r}}=$ contenido volumétrico de agua residual.

Además $\left(\sigma_{n}-u_{a}\right)=$ esfuerzo neto y $\left(u_{a}-u_{w}\right)=$ succión mátrica del suelo.

En este trabajo se presenta el comportamiento del esfuerzo cortante del suelo en estudio para distintos valores del grado de saturación (de succión), obtenidos mediante ensayes triaxiales (CD), los cuales se comparan con los resultados teóricos obtenidos mediante las ecuaciones de Bishop (1959), Fredlund et al. (1978) y Vanapalli et al. (1996).

\section{Materiales y métodos}

Los suelos que se han utilizado en esta investigación son de origen transportado y se obtuvieron en dos sitios; el primero en el lecho del río Verde a la altura de la comunidad "Cruz del Mezquite" en Ciudad Fernández, San Luis Potosí y el segundo se obtuvo en un banco de material de préstamo, localizado en el km 5+000 de la carretera Uriangato-Yuriria, en Guanajuato. El muestreo fue aleatorio y se practicó de forma alterada.

Con los suelos antes mencionados se elaboraron distintas mezclas y con cada una de ellas se fabricaron especímenes de suelo a los cuales se les practicaron ensayes de compresión simple de acuerdo con la norma ASTM D 2850-03a, con la intención de conocer el comportamiento de su resistencia al esfuerzo desviador para diferentes contenidos de agua. De esta serie de ensayes se determinó que una mezcla con una proporción de 30-70\% (de suelo de Ciudad Fernández y Uriangato, respectivamente), presentó un comportamiento favorable para esta investigación.

El propósito de estos ensayes fue comprobar que el suelo presentara un máximo en su resistencia al esfuerzo desviador a diferentes contenidos de agua en trayectoria de secado, esto para demostrar que la variación de los esfuerzos al corte, con respecto de la succión, no es

\section{Esfuerzo deviador-Contenido de agua}

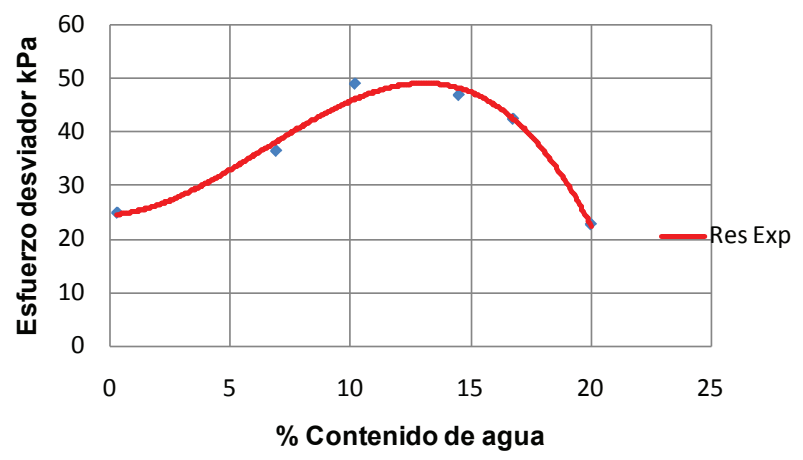

Figura 1. Comportamiento del esfuerzo desviador en función del contenido de humedad

lineal, como lo señalan Gan et al. (1988); Escario y Juca (1989), véase la figura 1.

Para identificar el suelo resultante se utilizó el Sistema Unificado de Clasificación de Suelos (SUCS) ASTM D 2487-00, para lo cual se practicó el ensaye granulométrico combinado (mallas e hidrómetro) ASTM D 422-63, límites de Atterberg ASTM D 4318-00. En forma adicional, se le determinó la contracción lineal, contracción volumétrica, ensaye proctor ASTM D 698-00 ${ }^{\varepsilon 1}$ y densidad de los sólidos, los resultados se presentan en la tabla 1.

Tabla 1. Propiedades índices de la mezcla de suelo

\begin{tabular}{lc}
\hline \multicolumn{1}{c}{ Propiedad } & Mezcla \\
\hline \% Grava & 0.00 \\
\% Arena & 68.82 \\
\% Limo & 29.41 \\
\% Arcilla & 1.78 \\
\% L. Líquido & N.P. \\
\% L. Plástico & 37.93 \\
\% I. Plasticidad & N.P. \\
SUCS & SM \\
\% Contracción Lineal & 1.99 \\
\% Contracción Vol. & 0.95 \\
P.V.S.M. kN/m ${ }^{3}$ & 17.31 \\
\% Contenido de agua & 21.2 \\
Densidad de los sólidos & 2.38 \\
\hline
\end{tabular}

En esta investigación se utilizó un suelo areno limoso $\mathrm{SM}$, cuyas características se muestran en la tabla 1. La distribución del tamaño de las partículas se presenta en la figura 2, en la cual se puede observar que las partícu- 


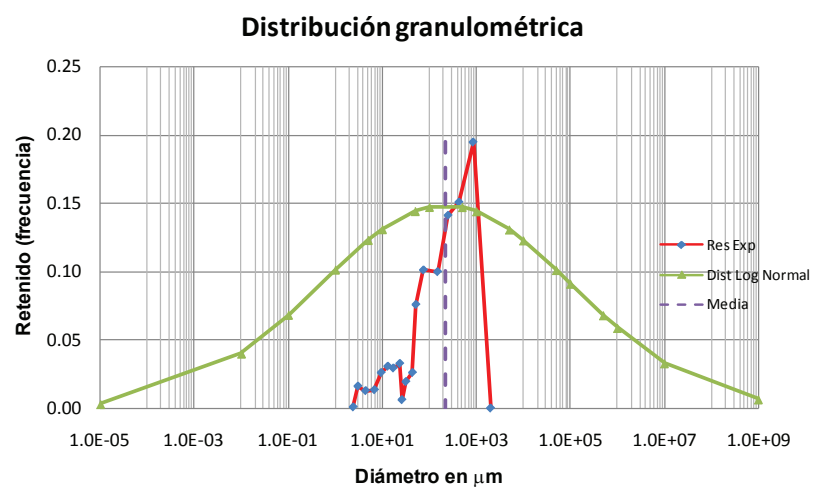

Figura 2. Distribución del tamaño de las partículas del suelo

las sólidas presentan aproximadamente una distribución logarítmica normal con diámetro medio de 221.31 $\mu \mathrm{m}$ y una desviación estándar de $429.27 \mu \mathrm{m}$.

Físicamente el suelo presenta el aspecto mostrado en la figura 3, esta imagen fue obtenida mediante el microscopio petrográfico, en la cual se observa que el suelo está formado por fragmentos angulosos y sub angulosos de roca volcánica con tamaño máximo de $2 \mathrm{~mm}$, también se localizaron fragmentos de cuarzo de origen volcánico, plutónico y metamórfico; así como fragmentos aislados de feldespato potásico y plagioclasas. Las partículas se muestran con un grado de alteración y oxidación moderado con algunos remanentes aislados de material vítreo.

Con este suelo se fabricó una serie de probetas cilíndricas de $3.60 \mathrm{~cm}$ de diámetro y $7.60 \mathrm{~cm}$ de altura, compactadas estáticamente para obtener un peso volumétrico, $\gamma m=18.01 \mathrm{kN} / \mathrm{m}^{3}$ con un contenido de agua, $\omega=21 \%$.

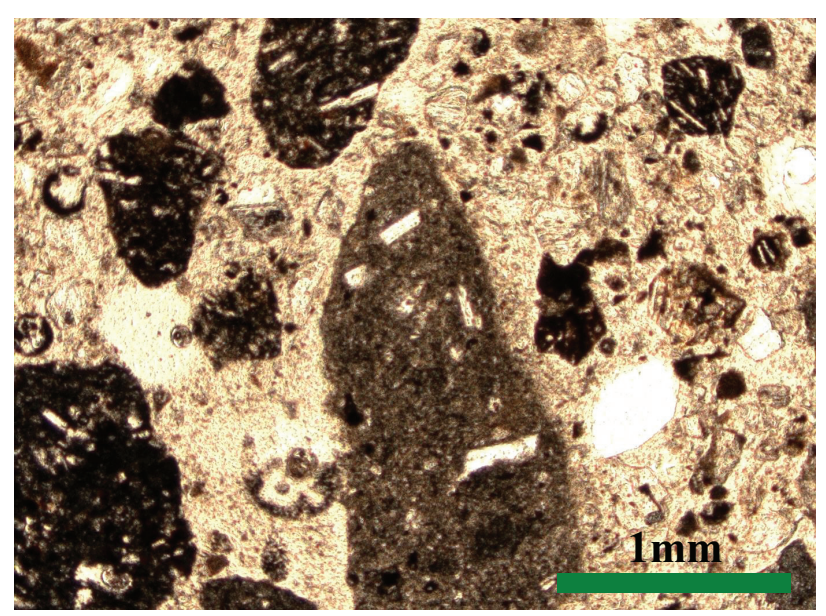

Figura 3. Imagen de la arena limosa utilizada en la investigación vista a $2.5 \mathrm{X}$
Todas las probetas fueron fabricadas en cinco capas. En cada capa se colocaron $30.50 \mathrm{~g}$ de suelo compactado mediante una prensa hidráulica hasta alcanzar el peso volumétrico previamente establecido. Además, entre cada una de las capas se tuvo la precaución de escarificar la superficie antes de agregar la siguiente capa y así lograr una muestra homogénea.

El lote de muestras fabricadas se dividió en dos partes, a una parte de las probetas se les incrementó la magnitud de la succión al reducir su contenido de agua por secado. De la misma manera, a la otra mitad de las probetas se les redujo su contenido de agua por secado hasta que alcanzaron la succión máxima. A partir de ahí, se inició un proceso de humedecimiento hasta que cada espécimen alcanzó el valor de succión previamente establecido.

Cuando los especímenes en trayectoria de secado y humedecimiento alcanzaron estas condiciones se les colocó dentro de una celda triaxial con succión controlada. La succión se indujo por medio de la circulación de vapor de agua con una humedad relativa seleccionada. La circulación del vapor se realizó por medio de una bomba peristáltica que trabaja a presión atmosférica y la humedad relativa se generó por medio de un recipiente que contiene agua con cierta concentración de sales. El esfuerzo de confinamiento aplicado a todas las probetas fue de $150 \mathrm{kPa}$. Al finalizar el ensaye se determinó la succión final de cada probeta por el método del papel filtro ASTM 529803.

Con la información obtenida a partir de los ensayes triaxiales (CD) practicados a las muestras de suelo, de acuerdo con la norma ASTM D 2850 03a, se determinó la variación del esfuerzo desviador a la falla, en función de la succión (grado de saturación).

\section{Resultados experimentales}

En forma simultánea durante la ejecución de los ensayes triaxiales $(\mathrm{CD})$ se obtuvo la curva característica del suelo en trayectoria de secado y humedecimiento. Para esto, una vez que los especímenes ensayados mediante el ensaye triaxial (CD) alcanzaron su esfuerzo máximo, los especímenes de suelo fueron retirados e instalados dentro de contenedores herméticos, buscando que no perdieran ni ganaran agua. Posteriormente, sobre cada espécimen se coloco un disco de papel filtro (utilizado como sensor) durante un periodo de 7 días, buscando que el papel filtro alcanzara el equilibrio de su contenido agua, con el contenido de agua del vapor del recipiente hermético. Cuando se alcanzó el equilibrio entre el agua del papel filtro y el vapor del recipiente, se le determinó a cada uno de los discos de papel filtro su 


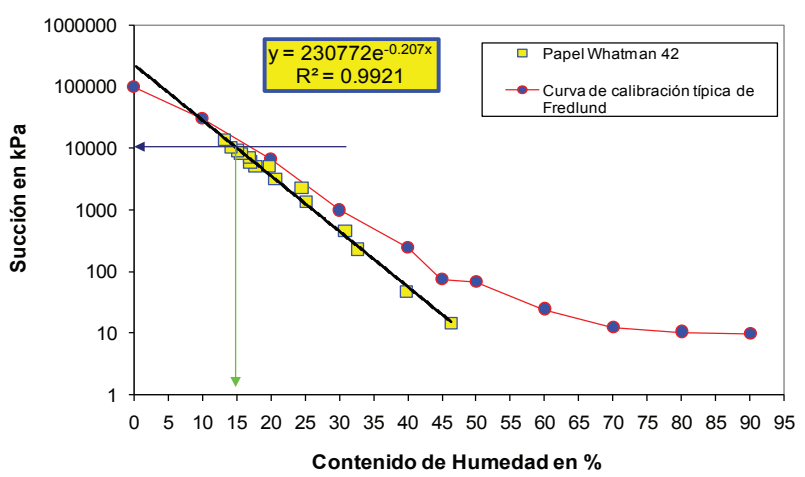

Figura 4. Curva de calibración del papel filtro Whatman 42

contenido de agua, según la norma ASTM D 221698. Una vez determinado el contenido de agua del papel filtro se utilizó la curva de calibración del papel filtro (figura 4) para inferir la magnitud de la succión del suelo a la falla.

El papel filtro se calibró previamente siguiendo el procedimiento que para este ensaye contempla la norma ASTM D 5298-03. Así mismo, se utilizó $\mathrm{NaCl}$ para proporcionar la succión osmótica a la solución. Los resultados de esta calibración se muestran en la figura 4.

Con la magnitud de la succión conocida y el grado de saturación para cada uno de los especímenes obtenido, en ambas trayectorias, se determinaron las curvas de retención del suelo en trayectoria de secado y humedecimiento que se muestran en la figura 5.

\section{Curva característica}

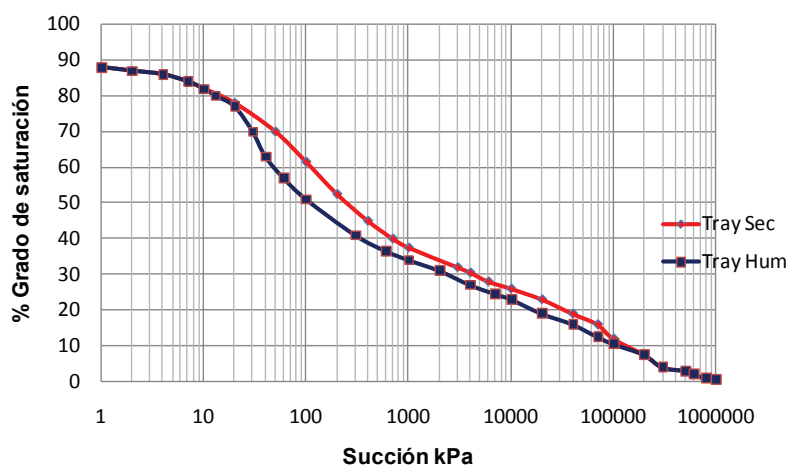

Figura 5. Curva característica del suelo, en secado y humedecimiento

En lo que respecta a los ensayes triaxiales (CD) se mencionó que los especímenes de suelo permanecieron en un proceso de equilibrio de la succión por un espacio de hasta cinco días, posteriormente se aplicó el esfuerzo isotrópico de $150 \mathrm{kPa}$ por un periodo de 24 horas, en seguida se aplicó el esfuerzo desviador a una velocidad de $0.001 \mathrm{~mm} / \mathrm{min}$ hasta alcanzar el esfuerzo máximo, los resultados de estos ensayes en ambas trayectorias se presentan en la figura 6 .

Con el esfuerzo desviador máximo obtenido de los ensayes triaxiales $(C D)$ practicado a cada uno de los especímenes en ambas trayectorias y el ángulo efectivo de fricción, se determinó el esfuerzo cortante experimental que el suelo presentó para cada grado de saturación. Se hace énfasis en que el ángulo efectivo de fricción del suelo se determinó a partir de un ensaye triaxial (CD) ASTM 2850 03a, practicado a especímenes de suelo en estado saturado y haciendo uso de la expresión siguiente:

$\phi^{\prime}=\operatorname{arcsen} \frac{\sigma_{1}-\sigma_{3}}{\sigma_{1}+\sigma_{3}}$

donde:

$\phi^{\prime}=$ ángulo efectivo de fricción

$\sigma_{1}=$ esfuerzo principal mayor

$\sigma_{3}=$ esfuerzo principal menor.

Además el esfuerzo cortante experimental se determinó con la siguiente expresión:

$\tau=\operatorname{sen} \phi^{\prime} \sqrt{\left(\frac{\sigma_{1}+\sigma_{3}}{2}\right)^{2}-\left(\frac{\sigma_{1}-\sigma_{3}}{2}\right)^{2}}$

donde:

$\tau=$ esfuerzo cortante

$\phi^{\prime}=$ ángulo efectivo de fricción

$\sigma_{1}=$ esfuerzo principal mayor

$\sigma_{3}=$ esfuerzo principal menor.

En la figura 6 se aprecia que las curvas de esfuerzo desviador en trayectoria de secado y humedecimiento presentan valores máximos que no son los mismos para ambas trayectorias.

Además, aquí se distingue que para grados de saturación pequeños el valor del esfuerzo desviador en trayectoria de secado es mayor que el obtenido en trayectoria de humedecimiento; sin embargo, para grados de saturación elevados se aprecia que el esfuerzo desviador en trayectoria de humedecimiento supera al obtenido en trayectoria de secado. No obstante, en ambas curvas se presenta un valor máximo.

Al comparar los esfuerzos cortantes teóricos obtenidos mediante el modelo de Bishop (1959) (figuras 7 y 8) es posible ver que los esfuerzos cortantes teóricos son casi en su totalidad inferiores a los obtenidos experimentalmente para succiones bajas, mientras que para succiones elevadas los esfuerzos cortantes teóricos au- 


\section{Esfuerzo desviador-Grado de saturación}

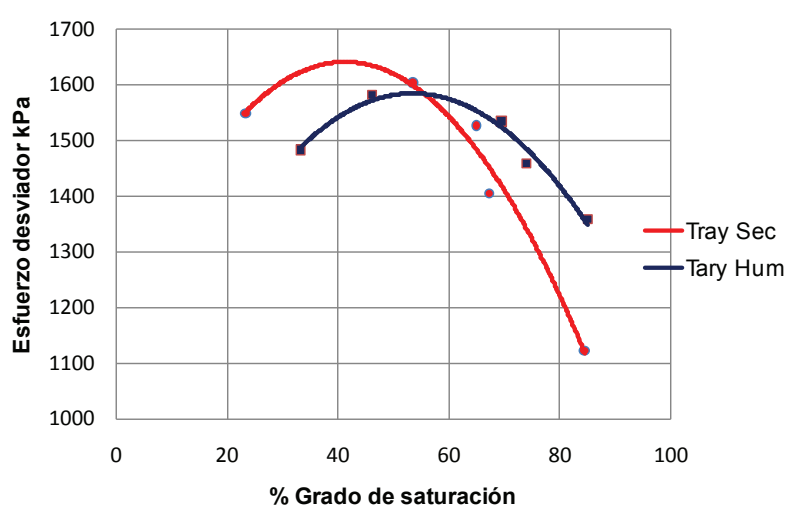

Figura 6. Esfuerzo desviador contra grado de saturación en secado y humedecimiento

mentan significativamente hasta llegar a un máximo después del cual decrecen.

En lo que respecta a los esfuerzos cortantes máximos obtenidos mediante el modelo de Fredlund et al. (1978) (figuras 7 y 8), se aprecia que para bajas succiones los esfuerzos cortantes teóricos resultan inferiores a los valores experimentales mientras que para succiones elevadas los esfuerzos cortantes teóricos son superiores a los experimentales.

Los esfuerzos cortantes pronosticados mediante el modelo de Vanapalli et al. (1996) son inferiores a los esfuerzos cortantes obtenidos experimentalmente para bajas succiones, no obstante, en succiones elevadas estos esfuerzos resultan ser superiores. Además, en las trayectorias de secado y humedecimiento, la magnitud de los esfuerzos cortantes decrece repentinamente al llegar a succiones elevadas.

Se puede apreciar en las figuras 7 y 8 que los resultados del modelo de Bishop (1959) guardan una tendencia más cercana a los resultados experimentales en ambas trayectorias, en comparación con los otros modelos. Por esta razón se propone el modelo de Bishop (1959), para analizar la resistencia de otros tipos de suelos cohesivos, como es el caso del suelo arcilloso de alta compresibilidad $\mathrm{CH}$, manejado en la aplicación práctica.

En la figura 9 se aprecia que los esfuerzos cortantes en trayectoria de humedecimiento obtenidos mediante el modelo de Bishop (1959) son hasta un $90 \%$ menor que los de los esfuerzos cortantes obtenidos experimentalmente. Sin embargo, los resultados obtenidos con los otros modelos son menos cercanos a los resultados experimentales, es decir, el modelo de resistencia para suelos no saturados que presenta resultados más cercanos al comportamiento del suelo es el modelo de
Bishop (1959). Por lo tanto, este modelo es el más recomendable para realizar el análisis de estabilidad del ejercicio del talud que se muestra más adelante.

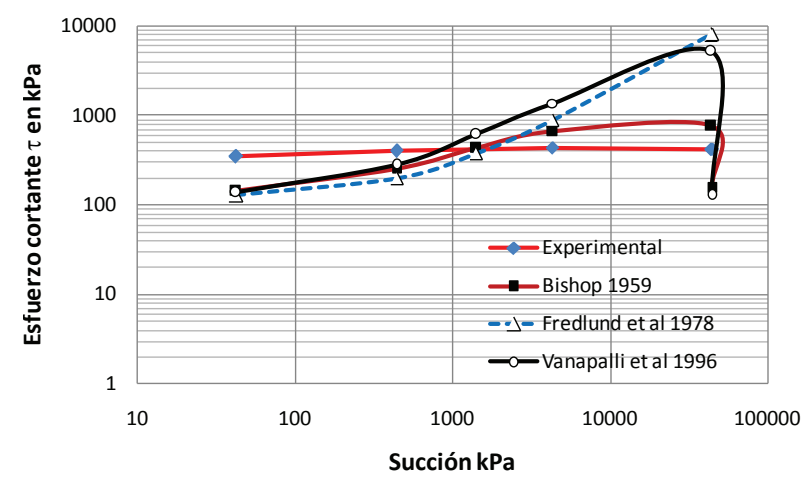

Figura 7. Curvas de esfuerzo cortante contra succión en secado

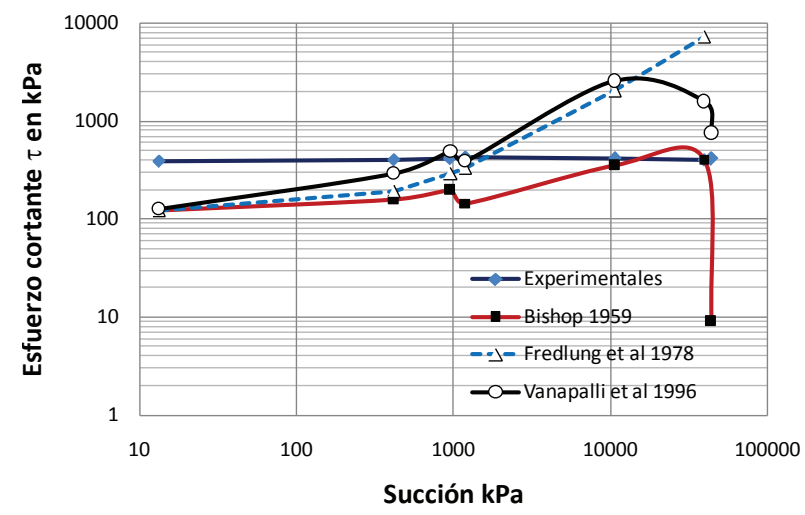

Figura 8. Curvas de esfuerzo cortante contra succión en humedecimiento

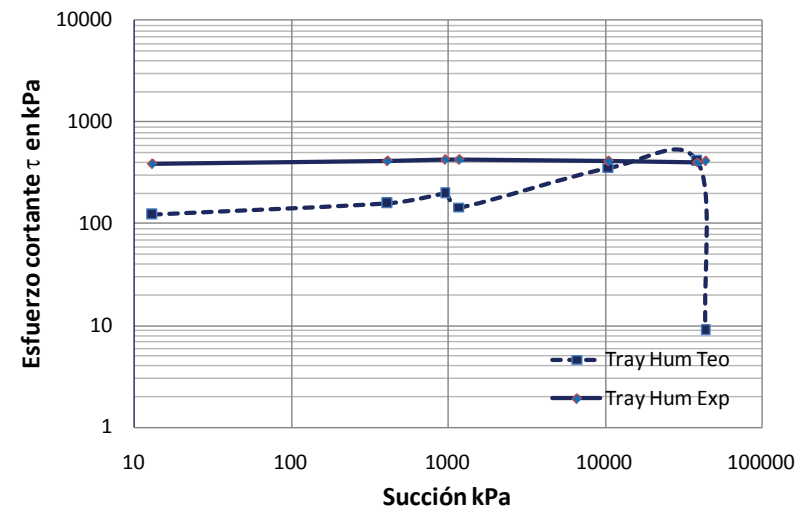

Figura 9. Esfuerzos cortantes contra succión, en trayectoria de humedecimiento, según el modelo de Bishop (1959) 


\section{Aplicación práctica}

Con el propósito de hacer una aplicación práctica del análisis de resistencia en suelos no saturados, se presenta el estudio de las condiciones superficiales de un talud de arcilla de alta compresibilidad $\mathrm{CH}$, que se encuentra en el km 240+000 sobre la "autopista del sol" a $20 \mathrm{~km}$ al norte de la ciudad de Chilpancingo y en las cercanías con el poblado Zumpango, en el estado de Guerrero. La figura 10 presenta una vista general de dicho talud.

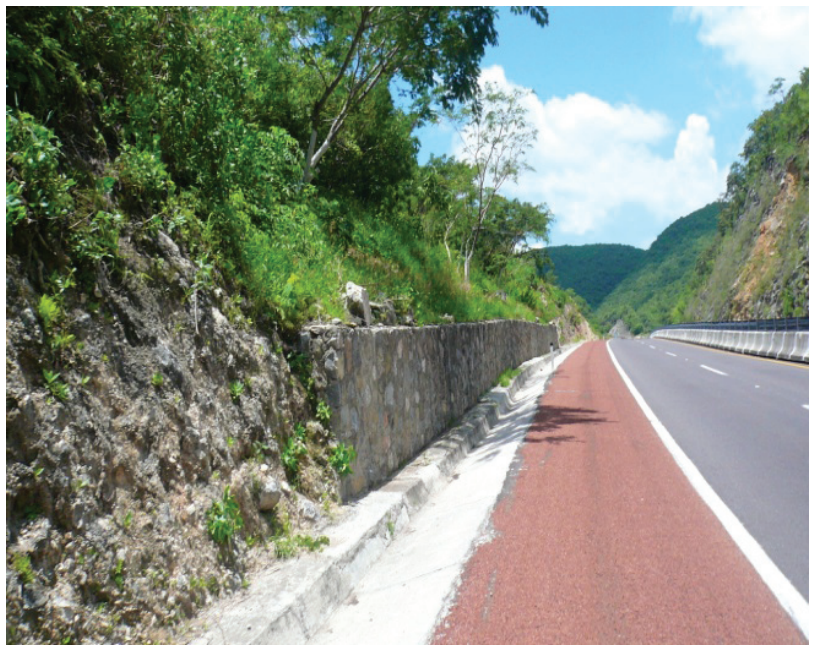

Figura 10. Aspectos del talud analizado

Desde que la autopista se puso en operación, dicho talud ha venido presentado problemas de estabilidad. Entre los factores principales de esta inestabilidad están las características litológicas, geométricas y mecánicas del talud, así como las condiciones climáticas de la región y el tipo de drenaje.

El tipo de clima en la zona de estudio va desde semiseco, semicálido hasta muy cálido, la temporada de lluvias se presenta normalmente de junio a septiembre con una precipitación media anual de 1,650 milímetros según lo refiere el IMT (2007).

La estabilidad de un talud depende en gran medida de la distribución de la presión del agua de poro interna, la cual depende a su vez, de la infiltración del agua de lluvia.

En el análisis de la estabilidad de este talud se tomaron en cuenta las variaciones de la resistencia del suelo debido a los cambios en el grado de saturación. Así, el análisis del talud se inició cuando el suelo presentó un grado de saturación cercano a cero, y terminó cuando el suelo alcanzó el estado saturado. El talud presenta una altura de $5.0 \mathrm{~m}$ y un ángulo de inclinación de, $\beta=31^{\circ}$.
Las condiciones naturales del suelo en campo son las que aparecen en la tabla 2.

Tabla 2. Propiedades del suelo in situ

\begin{tabular}{cccccc}
\hline$\omega$ & $\mathrm{e}$ & $\mathrm{n}$ & $\mathrm{G}_{\mathrm{w}}$ & $\gamma_{\mathrm{m}}$ & $\gamma_{\mathrm{d}}$ \\
\hline$\%$ & & $\%$ & $\%$ & $\mathrm{kN} / \mathrm{m}^{3}$ & $\mathrm{kN} / \mathrm{m}^{3}$ \\
44.10 & 1.38 & 57.91 & 82.69 & 15.35 & 10.64 \\
\hline
\end{tabular}

El análisis de la estabilidad del talud se realizó en trayectoria de humedecimiento. Es importante señalar que en la medida en la que el grado de saturación del suelo sufre modificaciones también lo hace la cohesión y el peso volumétrico. La información con la cual se desarrolló el análisis del talud para los distintos grados de saturación se muestra en la tabla 3.

Tabla 3. Variación de las propiedades mecánicas del suelo

\begin{tabular}{ccccc}
\hline $\mathrm{G}_{\mathrm{w}}$ & $u_{\mathrm{a}}-u_{\mathrm{w}}$ & $\gamma_{\mathrm{m}}$ & Cohesión & $\omega$ \\
\hline$\%$ & & $\mathrm{kN} / \mathrm{m}^{3}$ & $\mathrm{kPa}$ & $\%$ \\
100.00 & 0.27 & 16.35 & 0.00 & 53.33 \\
85.00 & 9.00 & 15.50 & 1.66 & 45.33 \\
71.00 & 40.00 & 14.70 & 2.37 & 37.87 \\
57.00 & 100.00 & 13.91 & 7.12 & 30.4 \\
27.00 & 1400.00 & 12.20 & 10.68 & 13.33 \\
0.00 & $1.00 \mathrm{E}+06$ & 10.68 & 12.26 & 0.00 \\
\hline
\end{tabular}

Para obtener los parámetros mecánicos del suelo se realizó una serie de ensayes triaxiales (CD) con muestras saturadas. De acuerdo con estos resultados, se determinó que el ángulo efectivo de fricción del suelo es $\phi^{\prime}=$ $22^{\circ}$. Además se desarrolló una serie de ensayes triaxiales (CD) con los cuales se pudo determinar la variación de la cohesión del suelo para distintos grados de saturación, a la vez, se determinó la magnitud de la succión (grado de saturación) que el suelo alcanzó al final del ensaye, los resultados se aprecian en la tabla 3. Con la información de la succión registrada (con grandes incrementos) en cada espécimen y con la magnitud del esfuerzo cortante (con incrementos menores) que el suelo presentó, se determinó que el ángulo de fricción aparente $\phi^{\mathrm{b}}=0.0023^{\circ}$. Con esta información se realizó el análisis del talud para los diferentes valores de la succión. El análisis del talud se realizó por el método de Bishop. Estos resultados aparecen en las figuras 11, 12, 13, 14, 15 y 16. 


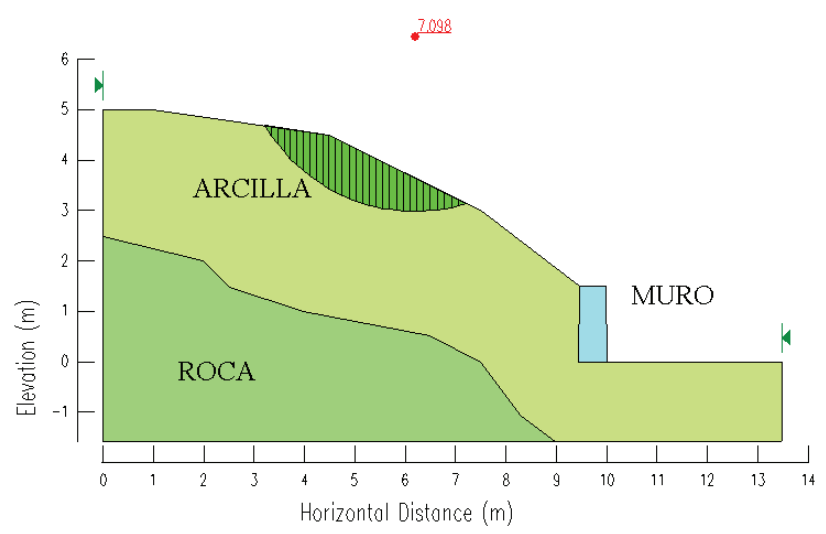

Figura 11. $F_{s}=7.098$ para un $G_{w}=0 \%$ y una $\psi=1000000 \mathrm{kPa}$

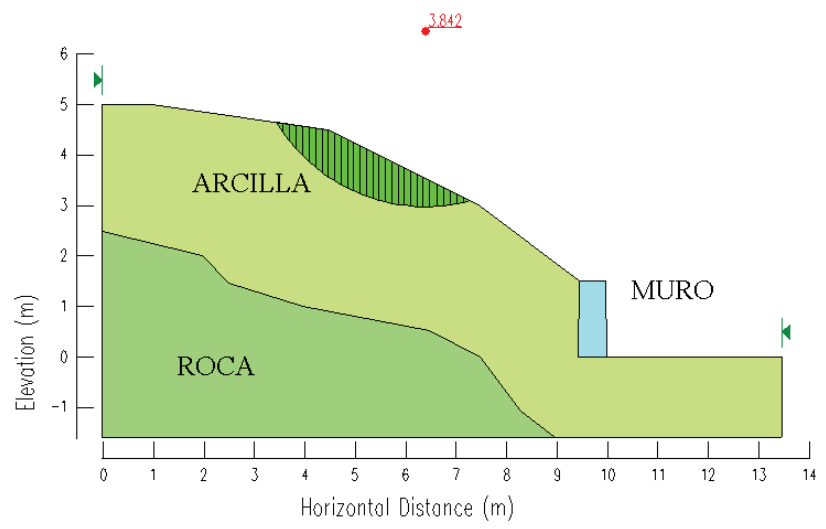

Figura 13. Fs $=3.842$ para un $\mathrm{G}_{\mathrm{w}}=57 \%$ y una $\psi=100 \mathrm{kPa}$

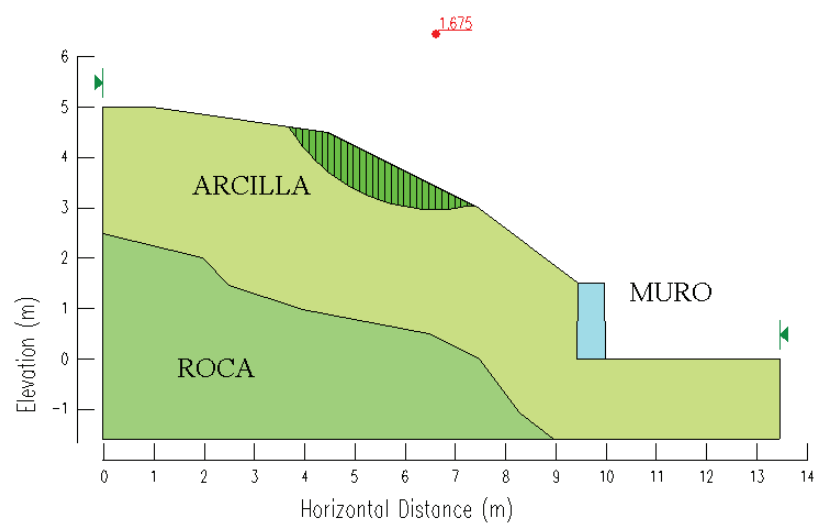

Figura 15. Fs $=1.675$ para un $\mathrm{G}_{\mathrm{w}}=85 \%$ y una $\psi=9.0 \mathrm{kPa}$

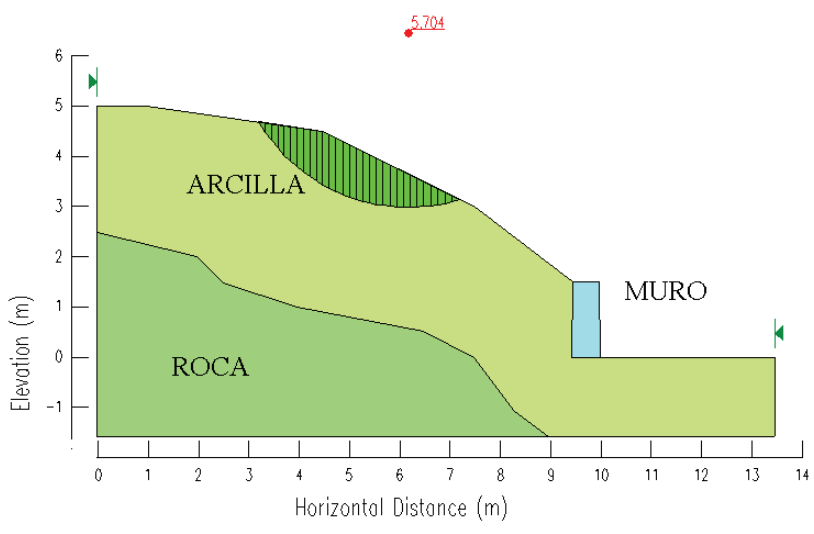

Figura 12. $\mathrm{Fs}=5.704$ para un $\mathrm{G}_{\mathrm{w}}=27 \%$ y una $\psi=1400 \mathrm{kPa}$

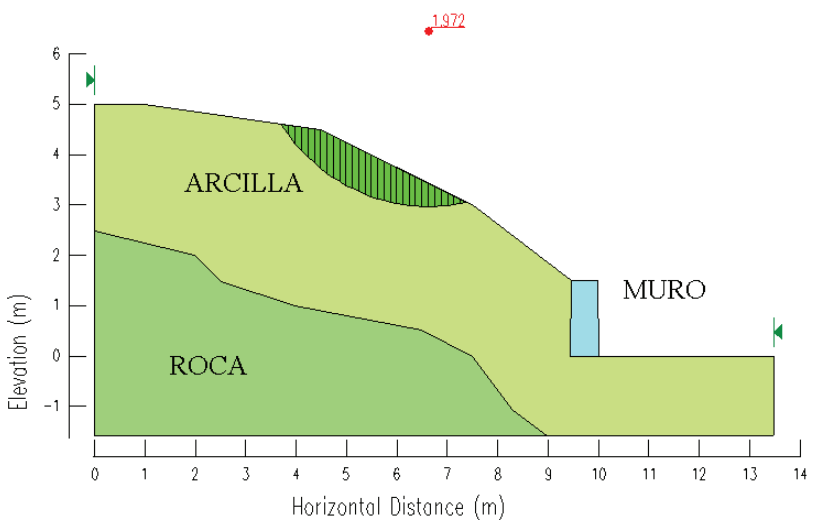

Figura 14. Fs $=1.972$ para un $\mathrm{G}_{\mathrm{w}}=71 \%$ y una $\psi=40 \mathrm{kPa}$

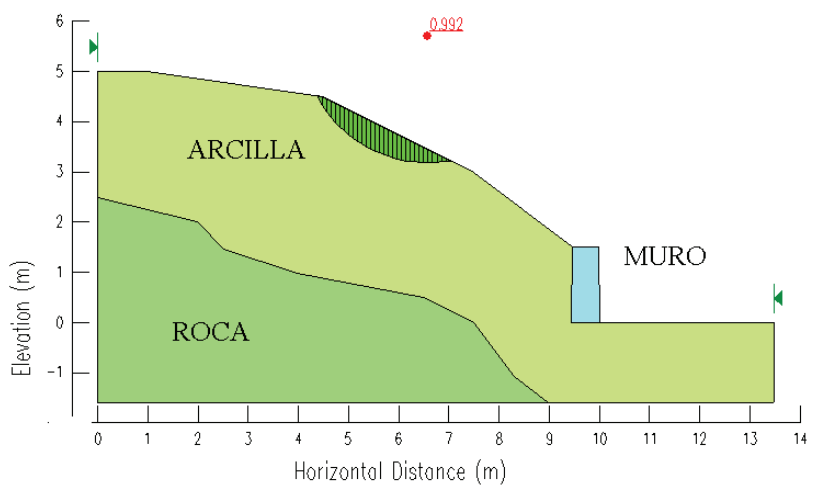

Figura 16. $\mathrm{Fs}=0.992$ para un $\mathrm{G}_{\mathrm{w}}=100 \%$ y una $\psi=0.27 \mathrm{kPa}$ 


\section{Variación del Factor de seguridad}

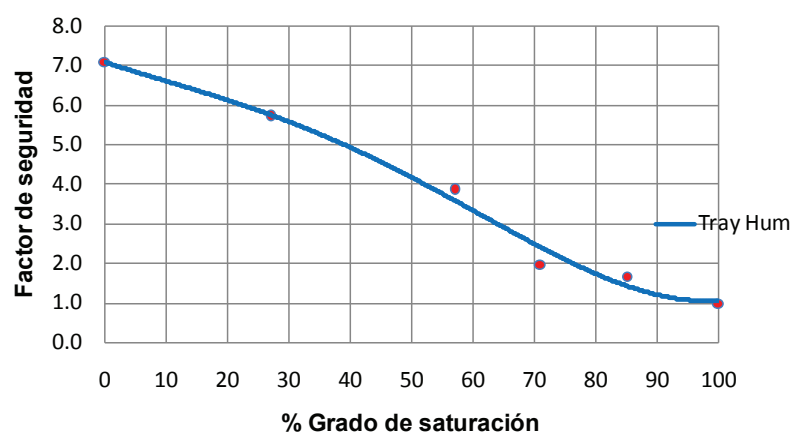

Figura 17. Variación del factor de seguridad en función del grado de saturación

Los resultados del análisis del factor de seguridad se muestran en la figura 17, en esta figura se observa cómo varía el factor de seguridad del talud en función del grado de saturación que el suelo experimenta. Del análisis de cada uno de los factores de seguridad se puede deducir que cuando el suelo alcance un grado de saturación de $90 \%$ se empezará a presentar riesgo de falla.

Para evitar que los taludes se humedezcan y lleguen a deslizarse es posible tomar medidas preventivas, por ejemplo, es posible colocar drenes y contracunetas que eviten al máximo el escurrimiento e infiltración de agua hacia el cuerpo del talud. En casos más severos es posible recubrirlos con vegetación o colocar mallas de acero conjuntamente con concreto lanzado o geotextiles plásticos.

\section{Conclusiones}

Parte experimental

- La variación de la resistencia al corte con el grado de saturación presenta un máximo en algunos suelos.

- El modelo teórico que más se aproxima a los datos experimentales analizados es el modelo de Bishop (1959).

- La resistencia del suelo se ve influenciada por la historia de humedecimiento y secado.

\section{Ejercicio práctico}

- El factor de seguridad de los taludes sujetos a humedecimiento, evoluciona con el tiempo.

- El talud analizado presenta riesgo de falla con grados de saturación cercanos a $90 \%$.
- Es posible elaborar mapas de riesgo por deslizamiento de los taludes y tomar medidas preventivas como mejorar el drenaje o proteger el talud.

\section{Referencias}

Bishop A.W. The Principle of Effective Stress. Teknisk Ukeblad, (número 39), 1959: 859-863.

Escario V., Juca J.F.T. Strength and Deformation of Partly Saturated Soils, en: Proc. 12th ICSMFE, Rio de Janeiro, volumen 1, 1989, 43-46 p.

Fredlud D.G., Morgenstern N.R., Widger R.A. The Shear Strength of Unsaturated Soils. Canadian Geotechnical Journal, volumen 15 (número 3), 1978: 313-321.

Fredlund D., Rahardjo H. Soil Mechanical for Unsaturated Soils, 1a ed., Nueva York, A Wiley-Intescience Publications, Inc, 1993.

Gan J.K.M., Fredlund D.G., Rahardjo H. Determination of the Shear Strength of Unsaturated. Soils. Can Geotech J, volumen 25, 1988: 500-510.

Rojas E., Rojas F. A Probabilistic Model for the Soil-Water Characteristic Curve, en: Proceeding of IV International Congress of Unsaturated Soils in Arizona, 2006.

Terzaghi K. The Shearing Resistance of Unsaturated Soils and the Angle Between the Planes of Shear, en: Proc. 1st Int. Conf. Soil Mech., volumen 1, 1936, 54-56p.

Vanapalli S.K., Fredlud D.G., Pufahl D.E., Clifton A.W. Model for the Prediction of Shear Strength with Respect to Soil Suction. Canadian Geotechnical Journal, volumen 33, 1996: 379-392.

\section{Bibliografía}

Cecilia A.C.C., Francisco E.M.G. y Mendoza A.D. Anuario estadístico de accidentes en carreteras federales 2006. Documento Técnico Núm 38. I.M.T. (S.C.T.), Sanfandila, Qro, 2007.

Alonso E.A.G., Loret A. y Delahaye C. Effect of Rain Infiltration on the Stability of Slopes, Unsaturated Soils, 1995.

Annual Book of ASTM STANDARDS. International Standard World Wide. Section four Construction Volume 04.08 Soil and Rock (I): D420-D-5611, 2004.

Bujang B.K.H., Ali-Faisal H., Rajoo R.S.K. Stability Analysis and Stability Chart for Unsaturated Residual Soil Slope. American Journal of Environmental Science, volumen 2 (número 4), 2006: 154-160.

Coulomb C.A. Essai sur une application des regles des maximis et minimis a quelques problemes de statique relatifs a l'architecture. Memoires de l'Academie Royale pres Divers Savants, volumen 7, 1776.

Fredlund D.G., Xing A. Equation for the Soil Water Characteristic Curve. Canadian Geotechnical Journal, volumen 31 (número 3), 1994: 521-532. 
Jian-Zhou, Jian-lin Y. Influences Affecting the Soil-Water Characteristic Curve. Journal of Zhejiang University Science, 2005.

Juárez B.E. y Rico R.A. Mecánica de suelos, tomo 1 y 2, XIV ed., México, Limusa, 1992.

Thamer-Ahmed M., Faisal-Hj A., Hashim S., Bujang B.K.H. Relationship Between Shear Strength and Soil Water Characteristic Curve of an Unsaturated Granitic Residual Soil.

American Journal of Environmental Sciences, volumen 2 (número 4), 2006:142-145.

Vanapalli S.K., Fredlund D.G. Comparison of Different Procedure to Predict Unsaturated Soil Shear Strength, Department of Civil Engineering, University of Saskatchewan, S.K. Canadá, S7N 5A9, 2000.

Whitlow R. Fundamentos de mecánica de suelos, 2a. ed., México, Compañía Editorial Continental, 1999.

\section{Este artículo se cita:}

\section{Citación Chicago}

Gallegos-Fonseca, Gustavo, Julio C. Leal-Vaca, Eduardo Rojas-González, René S. Mora-Ortíz. Efecto de la succión en la estabilidad de un talud de arena limosa. Ingeniería Investigación y Tecnología XIII, 03 (2012): 271-281.

\section{Citación ISO 690}

Gallegos-Fonseca G., Leal-Vaca J.C., Rojas-González E., MoraOrtíz R.S. Efecto de la succión en la estabilidad de un talud de arena limosa. Ingeniería Investigación y Tecnología, volumen XIII (número 3), julio-septiembre 2012: 271-281

\section{Semblanza de los autores}

Gustavo Gallegos-Fonseca. Es ingeniero civil, maestro en ciencias en la línea de mecánica de suelos y alumno del doctorado en ingeniería de la Facultad de Ingeniería de la Universidad Autónoma de Querétaro. Actualmente es profesor de la licenciatura en ingeniería civil de la UASLP ZM desde 1993 a la fecha.

Julio César Leal-Vaca. Es ingeniero civil, maestro en ciencias en la línea de mecánica de suelos y alumno del doctorado en ingeniería de la Facultad de Ingeniería de la Universidad Autónoma de Querétaro. Actualmente es profesor de la licenciatura en ingeniería civil de la U de G desde 1998 a la fecha.

Eduardo Rojas-González. Es profesor investigador de la Facultad de Ingeniería de la Universidad Autónoma de Querétaro.

René Sebastián Mora-Ortíz. Es alumno de la maestría en mecánica de suelos de la Facultad de Ingeniería de la Universidad Autónoma de Querétaro. 